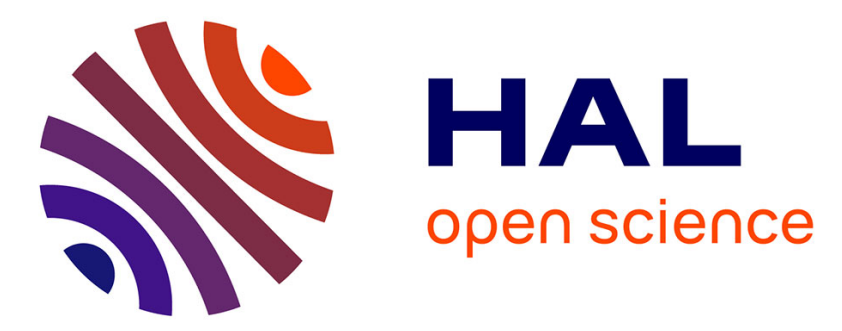

\title{
Adaptive Discrete Laplace Operator
}

Christophe Fiorio, Christian Mercat, Frédéric Rieux

\section{To cite this version:}

Christophe Fiorio, Christian Mercat, Frédéric Rieux. Adaptive Discrete Laplace Operator. ISVC 2011

- 7th International Symposium on Visual Computing, Sep 2011, Las Vegas, United States. pp.377-386. hal-00659019

\section{HAL Id: hal-00659019 \\ https://hal.science/hal-00659019}

Submitted on 12 Jan 2012

HAL is a multi-disciplinary open access archive for the deposit and dissemination of scientific research documents, whether they are published or not. The documents may come from teaching and research institutions in France or abroad, or from public or private research centers.
L'archive ouverte pluridisciplinaire HAL, est destinée au dépôt et à la diffusion de documents scientifiques de niveau recherche, publiés ou non, émanant des établissements d'enseignement et de recherche français ou étrangers, des laboratoires publics ou privés. 


\title{
Adaptive Discrete Laplace Operator
}

\author{
Christophe Fiorio ${ }^{1}$, Christian Mercat ${ }^{2}$, and Frédéric Rieux ${ }^{13}$ \\ 1 LIRMM, Université Montpellier 2, 161 rue Ada, \\ F-34392 MONTPELLIER, FranceIREM, S2HEP, Université Claude Bernard Lyon 1, \\ 43 bd du 11 Nov. 1918, \\ F-69622 Villeurbanne cedex \\ 2 I3M, Université Montpellier 2, c.c. 51 \\ F-34095 Montpellier Cedex 5, France
}

\begin{abstract}
Diffusion processes capture information about the geometry of an object such as its curvature, symmetries and particular points. The evolution of the diffusion is governed by the LAPLACE-BELTRAMI operator which presides to the diffusion on the manifold. In this paper, we define a new discrete adaptive Laplacian for digital objects, generalizing the operator defined on meshes. We study its eigenvalues and eigenvectors recovering interesting geometrical informations. We discuss its convergence towards the usual Laplacian operator especially on lattice of diamonds. We extend this definition to 3D shapes. Finally we use this Laplacian in classical but adaptive denoising of pictures preserving zones of interest like thin structures.
\end{abstract}

\section{Introduction}

Finding particular points on a discrete set is one of the most common problems in geometry processing applications. A particular example is to find a matching between pairs of shapes [12] and whether there exist isometric transformations between them. Another application is to find particular points that resist to a local deformation of the shape [14].

A large amount of work has been done in developing signature of a set defined by a digital mesh. Heat kernel or random walks have been widely used in image processing, for example lately by Sun, Ovsuanikov and Guibas [15] and Gebal, Bærentzen, Aanes and Larsen [5] in shape analysis.

In [15], a multi-scale signature was proposed, based on heat diffusion in order to detect repeated structure or information about the neighborhoods of a given points. This approach is connected to isometric matchings between pairs of shapes [13]. The heat kernel is also an isometric invariant, therefore studying it on each manifold, allows to compute a best matching map between the two shapes. In [1], a generalisation of diffusion geometry approach is proposed based on spectral distance.

The present article adapts to the digital geometry framework the properties of the Laplace operator on meshes. The main works in geometry diffusion [15, $5,13,1]$ are based on meshes shapes. We define as in those previous works a 
diffusion kernel on objects which are not meshes but digital objects made of a subset of $\mathbb{Z}^{2}$ (a set of pixels) or $\mathbb{Z}^{3}$ (a set of voxels).

In [4] an auto-adaptive digital process which captures information about the neighborhood of a point in a shape is introduced. They set up walkers on a digital object, and we observe the possibilities for them to walk along the discrete set. In this way, we compute weights corresponding to the time spent by a walker on each point of the shape.

This approach can be extended to 3 dimensional sets. We propose in this paper to study the relevance of this operator through the study of its eigenfunctions. The classical eigenfunctions of the Laplace-Beltrami operator are widely used in the mesh community to recover geometrical information about shapes $[11,7]$. For example in [7], Bruno Levy computes an approximation of these eigenfunctions to understand the geometry of manifolds.

This paper is organized as follows. First in Sec. 2, we describe an adaptive digital diffusion process on voxels and its associated Laplace operator. We extend this process on lattice of diamonds and we prove the convergence toward the usual Laplace operator in Prop. 5. Then in Sec. 3, we present two particular cases of use of this Laplacian, widely studied in the mesh community, in order to show its relevance. Finally in Sec. 4, we give another well known use of the diffusion as a classical convolution mask in gray-level images, to smooth and denoise. But the mask we use is our adaptive Laplacian and we give examples demonstrating that it preserves thin structures.

\section{Diffusion Processes}

\subsection{Heat Diffusion}

The heat kernel $k_{t}$ on a manifold $M$ maps a pair of points $(x, y) \in M \times M$ to a positive real number $k_{t}(x, y)$ which describes the transfer of heat from $y$ to $x$ in time $t$. Starting from a (real) temperature distribution $T$ on $M$, the temperature after a time $t$ at a point $x$ is given by a convolution of the initial distribution with the diffusion kernel:

$$
H^{t} f(x)=\int_{M} f(y) k_{t}(x, y) d y .
$$

The heat equation is driven by the diffusion process, the evolution of the temperature in time is governed by the (spatial) LAPLACE-BELTRAMI operator $\Delta_{M}: \frac{\partial f(t, x)}{\partial t}=-\Delta_{M} f(t, x)$ which presides to the diffusion on the manifold, just as random walks.

For a compact $M$, the heat kernel has the following eigen-decomposition:

$$
k_{t}(x, y)=\sum_{y \in M} e^{-t \lambda_{i}} \phi_{i}(x) \phi_{i}(y)
$$

where $\lambda_{i}$ and $\phi_{i}$ are the $i-t h$ eigenvalue and the $i-t h$ eigenfunction of the Laplace-Beltrami operator, respectively. 
The heat kernel $k_{t}(x, y)$, lately used by Sun, Ovsjanikov and Guibas [15], yields information about the geometry of the manifold. We have proposed in [4] a digital diffusion process which is adaptive to the geometry of a digital object. We defined a diffusion kernel similar to the continuous one just described and in this article we give examples of its use on $2 \mathrm{D}$ and $3 \mathrm{D}$ object.

\subsection{Auto-adaptive Process}

\section{Definition 1 (Adaptive Markov Chain).}

Let $\Sigma \subset \mathbb{Z}^{n}$ be a binary digital set, a sets of voxels. We define on $\Sigma$ the discrete time MARKOV chain whose states are voxels, and whose transition between two adjacent voxels is constrained by:

- Probability $\frac{1}{2^{n}}$ to move from the center of the voxels to one of its corners,

- Equiprobable repartition of the walkers from a corner to its incident voxels.

To illustrate the definition we propose an example on a $2 \mathrm{D}$ set (Fig. 1). We set up 24 walkers on the gray pixel to get an integer number of walkers in each pixel.

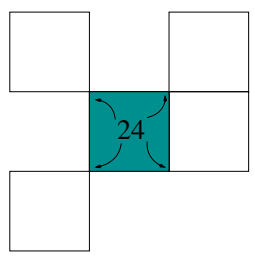

(a) From pixel to corners.

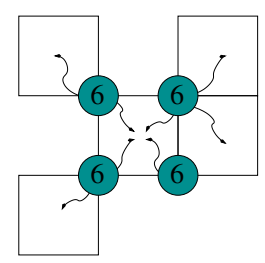

(b) From corners to adjacent pixels.

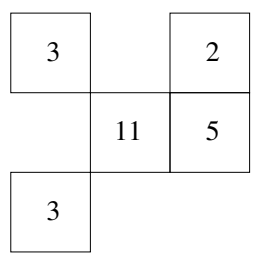

(c) Final mask after 1 step.

Fig. 1: Diffusion on an irregular set.

Remark 2. This standard choice amounts to walkers with no memory and only local knowledge, the celebrated short-sighted drunken man. We note this process $A_{s}^{m}$ for a walker starting at any given point, with $m$ the number of iterations of the process.

The 1-step MARKov process transition matrix $A_{s}$ is simply a weighted version of the adjacency matrix of the digital object $\mathcal{M}$. We note $u_{0}$ the distribution of walkers on the digital object at time 0 . We call $X_{m}$ the MARKov chain defined by Def. 1 iterated $m$ times and $u(m, x)$ the number of walkers at $x$ after $m$ steps starting from $u_{0}$ that is the expectation as a convolution of the initial distribution with the MARKOV kernel:

$$
u(m, x)=\mathbb{E}_{x} u_{0}\left(X_{m}\right)=\sum_{y \in \mathcal{M}} u_{0}(y) A_{s}^{m}(x, y) .
$$


The evolution of this expectation follows

$$
\begin{aligned}
\frac{u(m+1, x)-u(m, x)}{2} & =\sum_{y \in \mathcal{M}} u_{0}(y)\left(A_{s}^{m}\left(A_{s}-I d\right)\right) \\
& =\left(A_{s}-I d\right) u(m, x)
\end{aligned}
$$

Analogous to the case of the continuous heat diffusion, the diffusion equation reads

$$
\frac{\Delta u(m, x)}{\Delta m}=\left(A_{s}-I d\right) u(m, x)
$$

We therefore define the discrete Laplacian $\Delta_{M}:=-\left(A_{s}-I d\right)$

Property 3. On $\mathbb{Z}^{2}$, the diffusion leads to the Gaussian binomial masks $A_{s}^{m}(x, y)=$ $\left(\begin{array}{c}m \\ |x-y|\end{array}\right)$

This property was proven in [4]. The convergence of the Laplacian to the continuous one on the square lattice is a particular case of Prop. 5 on a lattice of diamonds.

\subsection{Generalization to Lattice of Diamonds}

In the previous section we defined a diffusion model on square pixels, with diagonal ratio equal to one. This model can be easily extended to quadrilaterals with a diagonal ratio equal to a more general value $\rho$, leading to a generalization of (Def 1) and similar convergence results.

Definition 4. Let $\Sigma$ be a sets of quadrilaterals of horizontal diagonal ratio $\rho$, that is to say a lattice of diamonds. We define on $\Sigma$ the discrete time MARKOV chain where the states are the quadrilaterals, and the transition between two quadrilaterals is constrained by:

- Probability $\frac{\rho}{2 \rho+\frac{2}{\rho}}$ to move from the center of a pixel to its two horizontal corners and $\frac{\frac{1}{\rho}}{2 \rho+\frac{2}{\rho}}$ to the vertical ones.

- Repartition on the incident quadrilaterals weighted by the distance, $\rho$ or 1 from the corner to the center of the neighbor.

Proposition 5. Let $\Sigma$ be a set of quadrilateral of horizontal diagonal ratio $\rho$. Then $\Delta_{M}$ converges toward the usual Laplace operator.

On irregular shapes, uneven adjacency between voxels produces irregular diffusion due to curvature. A similar convergence on irregular lattices has been proved in [10] on discrete conformal structures. We foresee that a similar proof will be possible for the current definition of the Laplacian and it will be the subject of future work. Moreover in the next section, we propose examples to show the relevance of the operator on irregular structures, to recover information about the geometry of shapes. 


\section{Application of The Laplace Operator on 2D and 3D discrete objects}

In this section we propose an application of the discrete Laplacian on 2D and 3D discrete objects. In [7], Bruno Levy uses the eigenfunctions of the LaplaceBeltrami operator $\Delta=\partial^{2} / \partial x^{2}+\partial^{2} / \partial y^{2}$ of the considered object to understand its geometry or its topology. These eigenvectors are proven to be noise resistant and a cut-off in frequency provides interesting unsupervised segmentations. A similar idea is proposed in [15], with the heat kernel signature (HKS) of a digital shape. HKS is a natural multi-scale characterization of the neighborhood of a given point $x$. We construct a similar signature for pixel or voxel discrete shapes and show on examples that they capture information of the global geometry: given the spectrum $\operatorname{sp}(\Delta)=\left\{\lambda_{i}\right\}$ and eigenvectors $\Delta \phi_{i}=\lambda_{i} \phi_{i}$, we construct the Heat Kernel Signature

$$
k_{m}(x, y)=\sum_{y \in M} e^{-m \lambda_{i}} \phi_{i}(x) \phi_{i}(y)
$$

\subsection{Segmentation}

Eigenvectors of the Laplacian, because of their interpretation as vibration modes and robustness to noise, have been widely used and documented in the mesh community for unsupervised clustering of protrusions and limbs segmentation $[7,9]$. The first eigenvectors, associated with highest eigenvalues, correspond to different "breathing" or "vibrating" modes, so that positive and negative value zones segment the object in meaningful regions. We give some examples (see Fig. 2c) of this use in the digital setup.

\section{$3.2 \quad$ Heat Kernel Signature}

We propose in this subsection an application of the eigenvectors of the Laplacian previously defined, as a digital signature of each point. This signature called HKS [15], has been applied with a version of the Laplacian operator on Meshes. We propose as an example of application the same operator based on our operator on voxels in Fig. 3.

\section{Gray Level Diffusion: Application to Denoising}

An application of this discrete version of the Laplacian is image denoising. The search for efficient image denoising algorithms is still active and related works on the subject is important. The main classical linear filtering used is the Gaussian kernel proposed in [8]. This kernel is optimal in regular parts but edges are blurred. Several methods are introduced in [2], to limit this blurring effect. An anisotropic weighted average to reduce the intensity of noise is proposed in [3]. 


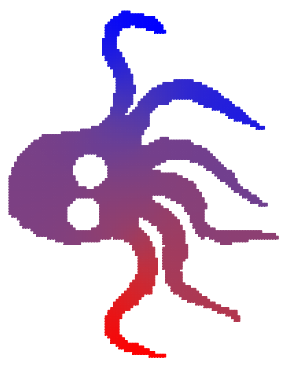

(a) First Eigenvectors on the octopus

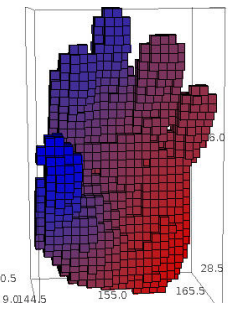

(d) Second Eigenvectors on the $3 \mathrm{D}$ hand

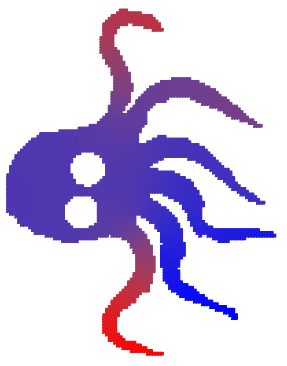

(b) Second Eigenvector on the octopus

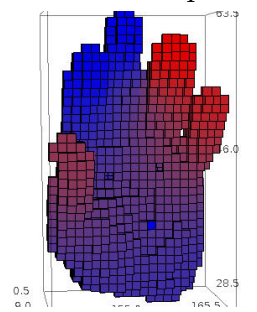

(e) Third Eigenvector on the 3D hand

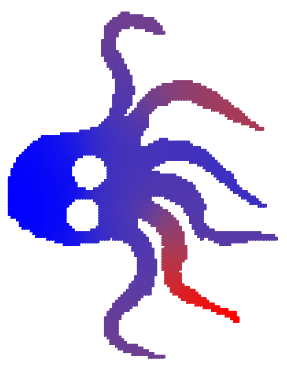

(c) Fourth eigenvector on the octopus

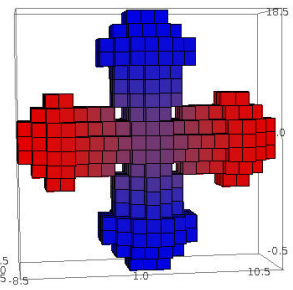

(f) Third eigenvector of a $3 \mathrm{D}$ star

Fig. 2: Eigenvectors of the Laplacian on an octopus. The eigenvectors can be interpreted as vibration modes, each one of the first eigenvectors being associated with different tentacles of the octopus. A similar analysis could be done on 3D digital object, like a hand or digital star.

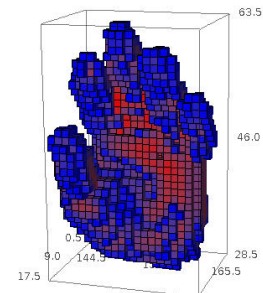

(a) Heat Kernel signature (HKS) computed on digital hand.

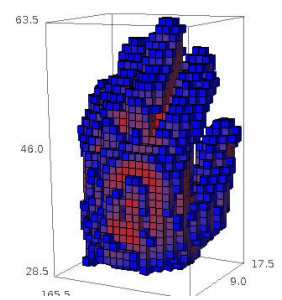

(b) Other view of the hand with HKS.

Fig. 3: On this figure, we have computed the HKS according to our definition of the Laplace operator. In blue the point of maximal curvature have been found efficiently, in red the point with low curvature. We map the hand with the values of the HKS for a given time $m$. 
They are based on extrinsic Gaussian filters while ours is adaptive to the digital object, converging to a Gaussian filter in the isotropic case:

We propose to define a Discrete Time MARKov Chain on a gray-level image. The idea is to let a walker wander on a discrete image with a gray level intensity that represents a hilly landscape. We use the previous pixel to pixel transition, for different thresholds, weighted by the gray level (understood as an interest map) of adjacent pixels:

We consider high gray values as high diffusion directions, that is to say the walker prefers to climb up on the highest value of its neighborhood.

Let $\left\{g_{1}, g_{2}, \ldots g_{8}\right\}$ the sorted gray intensity values of the 8-neighbors of a given pixel $p_{i}$ with $g_{1} \leq g_{2} \leq \ldots \leq g_{8}$. We note $p_{i}^{(j)}$ the $j$-th neighbors of $p_{i}$ with the gray level value $g_{j}$.

We construct iteratively the convolution mask for the 8-neighborhood of $p_{i}$. At each iteration we look for the neighbors of $p_{i}$ above the current threshold, we compute its diffusion mask, then we multiply the transition probability by the smallest gray intensity in the set and we delete this pixels from the neighborhoods, updating the threshold to the next lowest value. We continue thinning the neighborhood until there are no more pixels. If all the values are equal, we only do one iteration. If all the value are different we must build eight different masks among the $2^{8}$ possible. We note $\theta_{i}$ the number of gray intensity values that are different. We note $A_{s}^{(k)}$ the $k$-th matrix of transition of the set (this is the transition matrix where at least $k$ neighbors have been deleted). Then the final values of the masque is given by: $\operatorname{Mask}(i)=\sum_{k=1}^{\theta_{i}} A_{s}^{(k)}(i) g_{k} / \sum_{k=1}^{\theta_{i}} g_{k}$

Property 6 (Regular Gray Mask).

Let $\Sigma$ be a gray level set of pixels. If the $\left\{g_{0}, g_{1}, g_{2}, \ldots g_{8}\right\}$ are all equal (with $g_{0}$ the gray value of $p_{i}$ ), the the final mask is a classical Gaussian mask.

Proof. We have all the gray intensity values that are equal, then $\theta_{i}=1$. Then the Mask centered on the pixel $p_{i}$ is only:

$$
\operatorname{Mask}(i)=\frac{A_{s}(i) g_{0}}{g_{0}}
$$

by Property $3, \operatorname{Mask}(i)$ is a Gaussian mask.

The aim of this construction is to build convolution masks that are adaptive to gray level images. But on regular colors intensities we want to convolve a pixel with a mask that only depends on its distance to neighbors (Property 6)

The final convolution mask can be also seen as the 1 step transition probability of a Discrete Time MARKov Chain starting from the center to the neighbors.

This diffusion allows a walker wandering on an image to diffuse faster in the highest gray values of the neighborhood. This is useful when the user, or statistical analysis, provides an interval of gray values selecting zones of the object which are likely to be of interest, or when an interest function, such as the 


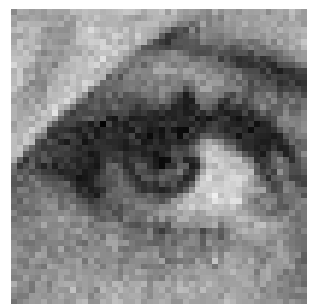

(a) Original image of Lena with Gaussian noise

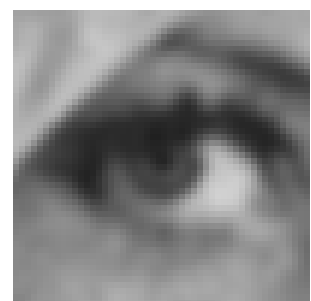

(b) Classical convolution with a Gaussian mask.

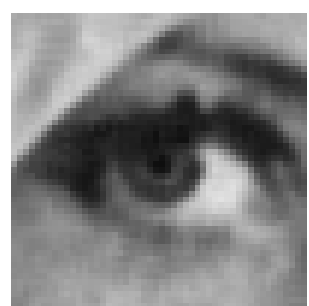

(c) Convolution with the gray-level adaptive mask.

Fig. 4: An example of application of the adaptive gray masks of convolution on a noisy version of Lena. We compare the noise reduction with the classical Gaussian mask. Clearly the blurred effect is less important in the convolution with our adaptive mask, contours are highlighted by a sharper contrast and reduced noise.

contrast (see Fig. $5 \mathrm{~g}$ ) is given as a gray level map of interest. We then compute the adaptive convolution mask for this interest level "picture" and apply it to the original image. This way, zones of similar interest (whether high or low) are smoothed out as with a regular (adaptive) Gaussian mask, but zones of different interest levels are not as mixed, the diffusion taking place mostly along the level sets of constant interest, therefore preserving, or even enhancing the thin structures.

Some results are shown in Fig. 4a, Fig. 5a and Fig. 5d. Those images are noisy and we chose a particular information to preserve. In the case of the peacock, we want to preserve the thin feathers of the bird, therefore selecting by statistical analysis, the range of intensities associated with the feathers as higher interest zones. A Gaussian mask would blur the feathers while our adaptive mask preserves them. For Lena, in Fig. 4c, we applied the mask of the noisy image on itself, and the result is an image which is less blurred with a reduction of the noise. We can compare the final result with an application of a Gaussian mask Fig. 4b

\section{Conclusion}

We have described a diffusion process on a digital object made of pixels or voxels, defined as a random walk on adjacent cells, generalizing diffusion on meshes. This process allows us to define a new discrete adaptive Laplace operator. We proved that this operator converges toward the usual continuous Laplace operator on diamond lattices. As in recent works on heat kernel spectral analysis for the Laplacian on meshes, we studied some properties of its eigenfunctions on particular objects and showed that we recover information about the geometry such as unsupervised segmentation or feature points detection. We have 


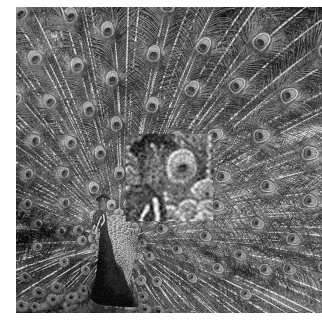

(a) A noisy image of a peacock.

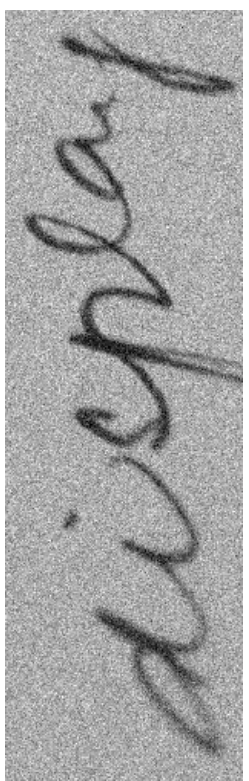

(d) Scanned text page

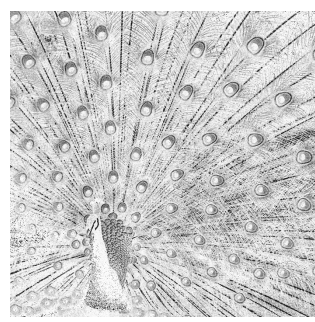

(b) Shape of interest: feathers of the peacock.

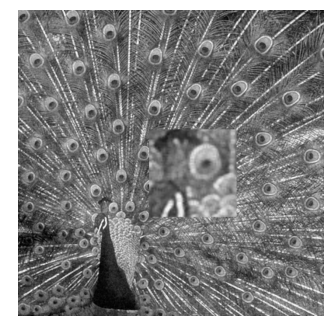

(c) Convolution with the gray-level adaptive mask.

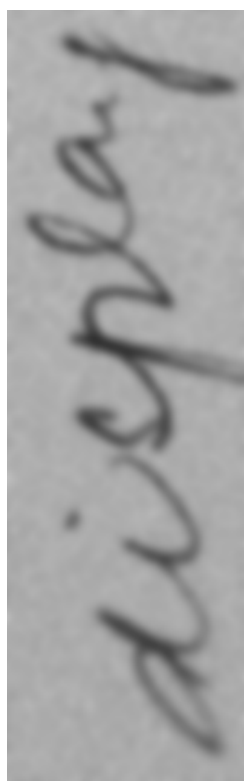

(e) Convolution using Gaussian

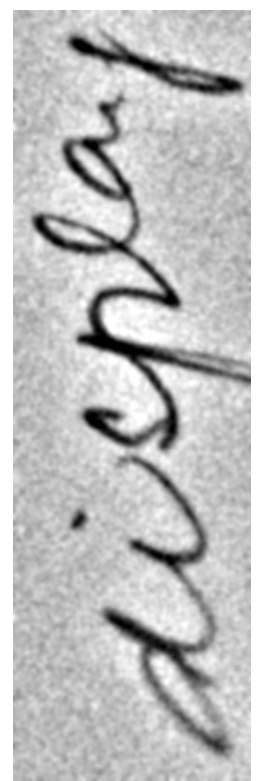

(f) Convolution using Fast Fourrier Transform bandpass filter

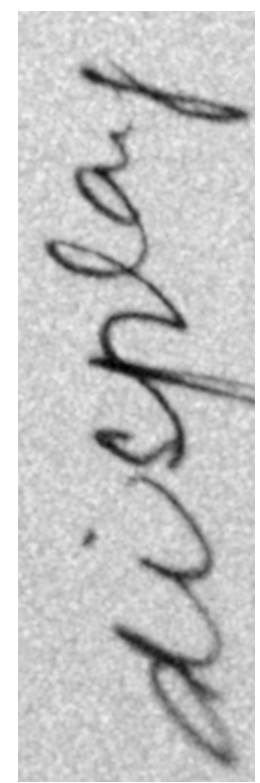

(g) Convolution using Adaptative convolution mask

Fig. 5: An example of application of the adaptive gray masks of convolution on noisy image of peacock, and on letters. On those images, we want to preserve or enhance particular information, for example defined by a certain gray level range. For example in (5b) we want to preserve the structure of the feathers. With a classical Gaussian mask, the fine structure is erased by the convolution, here its not the case, smoothing is performed along the structures. Notice also the preservation of the eye of the feathers despite the convolution. On Fig. 5d, the original image is noisy. Fig. $5 \mathrm{~g}$ reveals the contour, after the convolution, preserving the structures of the letters. We compare the adaptive convolution to classical gaussian and bandpass filter using fast Fourier transform. 
used this adaptive Laplacian on grey level images to smooth and denoise images while preserving regions or features of interest such as thin tubular structures. This work can be transposed to non binary $3 \mathrm{D}$ images and will be the subject of future work.

\section{References}

1. Michael M. Bronstein and Alexander M. Bronstein. Shape recognition with spectral distances. IEEE Trans. Pattern Anal. Mach. Intell., 33(5):1065-1071, 2011.

2. Antoni Buades, Bartomeu Coll, and Jean-Michel Morel. Image denoising methods. a new nonlocal principle. SIAM Review, 52(1):113-147, 2010.

3. Antoni Buades, Bartomeu Coll, and Jean-Michel Morel. Self-similarity-based image denoising. Commun. ACM, 54(5):109-117, 2011.

4. Christophe Fiorio, Christian Mercat, and Frédéric Rieux. Curvature estimation for discrete curves based on auto-adaptive masks of convolution. In Reneta P. Barneva, Valentin E. Brimkov, Herbert A. Hauptman, Renato M. Natal Jorge, and João Manuel R. S. Tavares, editors, CompIMAGE, volume 6026 of Lecture Notes in Computer Science, pages 47-59. Springer, 2010.

5. Katarzyna Gebal, J. Andreas Bærentzen, Henrik Aanæs, and Rasmus Larsen. Shape Analysis Using the Auto Diffusion Function. In Konrad et al. [6], pages 1405-1413.

6. Polthier Konrad, Alexa Marc, and Kazhdan Michael, editors. Symposium on Graphics Processing. Eurographics Association, 2009.

7. Bruno Lévy. Laplace-beltrami eigenfunctions towards an algorithm that "understands" geometry. In SMI, page 13. IEEE Computer Society, 2006.

8. Michael Lindenbaum, M. Fischer, and Alfred M. Bruckstein. On gabor's contribution to image enhancement. Pattern Recognition, 27(1):1-8, 1994.

9. Diana Mateus, Radu Horaud, David Knossow, Fabio Cuzzolin, and Edmond Boyer. Articulated shape matching using laplacian eigenfunctions and unsupervised point registration. In CVPR. IEEE Computer Society, 2008.

10. Christian Mercat. Discrete Riemann surfaces and the Ising model. Comm. Math. Phys., 218(1):177-216, 2001.

11. N. Nadirashvili, Dzh. Tot, and D. Yakobson. Geometric properties of eigenfunctions. Uspekhi Mat. Nauk, 56(6(342)):67-88, 2001.

12. M. Ovsjanikov, Q. Mérigot, F. Mémoli, and L. Guibas. One point isometric matching with the heat kernel. In Eurographics Symposium on Geometry Processing (SGP), volume 29, 2010.

13. Maks Ovsjanikov, Quentin Mérigot, Facundo Mémoli, and Leonidas J. Guibas. One point isometric matching with the heat kernel. Comput. Graph. Forum, 29(5):15551564, 2010.

14. Raif M. Rustamov. Laplace-beltrami eigenfunctions for deformation invariant shape representation. In Alexander G. Belyaev and Michael Garland, editors, Symposium on Geometry Processing, volume 257 of ACM International Conference Proceeding Series, pages 225-233. Eurographics Association, 2007.

15. Jian Sun, Maks Ovsjanikov, and Leonidas Guibas. A Concise and Provably Informative Multi-Scale Signature Based on Heat Diffusion. In Konrad et al. [6], pages $1383-1392$ 